\title{
Medication Adherence of Elderly Citizens in Retirement Homes Through A Mobile Phone Adherence Monitoring Framework (Mpamf) for Developing Countries: A Case Study in South Africa
}

\author{
Alfred Coleman \\ School of Computing, University of South Africa, South Africa
}

\begin{abstract}
Background: This paper investigated medication adherence among elderly people in retirement villages in South Africa. A case study approach was used. Methods: Twelve participants were purposively selected from six retirement homes in Gauteng Province. Data collected used semi-structured open ended interview questions. Interviewees (elderly people above 60 years) were asked to tell in their own words different types of medicine taken daily, how many times, how often they forgot to take their medication every week, and how often someone reminded them to take their medication. Interviewees were further asked if they owned mobile phones. Results: Findings revealed that elderly people take an average of four-five pills three times daily for chronic diseases like coronary artery. Findings further revealed that $100 \%$ of elderly people own mobile phones. However, they received no reminders when they forgot their medication. Conclusion: This led to a proposed Mobile Phone Adherence Monitoring Framework (MPAMF) based on self-efficacy of elderly people. MPAMF could leverage mobile phones technologies to remind elderly people to take their medication as prescribed, warn them when they take medication at incorrect times and report to a caregiver when they constantly miss medication event.
\end{abstract}

Keywords: Medication, Adherence, Elderly citizen, Retirement home, Mobile phones

\section{INTRODUCTION}

The World Health Organization (WHO) indicates that only about $50 \%$ of patients take their medicines as prescribed. ${ }^{1}$ The remaining $50 \%$ do not take their medication as prescribed. WHOconsiders this to be a poor adherence rate and has published an evidence-based guide for health care providers, health care managers, and policymakers aimed at improving strategies for medication adherence. ${ }^{2} \mathrm{~A}$ recent survey commissioned by the National Community Pharmacists Association (NCPA) also reported that nearly three out of every four American patients do not take their prescribed medicine as directed. ${ }^{3}$ The issue of non-adherence in American is mirrored in South Africa Society. The problem of poor adherence or non-adherence is exacerbated among elderly citizens who live in retirement homes or retirement villages in South Africa.Retirement homes and villages in South Africa are characterised by the presence of older people who no longer work, and who are over 60 years of age. In this research paper the term "retirement homes" will be used.

According to Kinsella and Phillips the proportion of the older population $(>65)$ in South Africa was over 2.47 million in 1985 and this will increase exponentially to 5.23 million in $2025 .{ }^{4}$ Such an increase in the
DOI: 10.5530/ijper.48.3.2

Address for correspondence: Dr. Alfred Coleman School of Computing, University of South Africa, South Africa

Phone: 0027116709108 Mobile: 0027731370859

E-mail: colema@unisa.ac.za

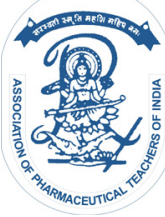

www.ijper.org 
older population of any countryaffects various social and economic structures of society,resulting in a lackof intergenerational support systems, workplace pension funds, social welfare assistance, health insurance/medical aid funds, and health-care provision. ${ }^{5}$ This economic problem becomes worse when members of the older population do not adhere to their prescribed medication intake. It has been estimatedthat medication nonadherence rates in the elderly range from $40 \%$ to as high as $75 \% .^{6}$

The problem of medication non-adherence among the elderlyin retirement homes results in higher rates of hospital admissions than non-adherence by the general populationdoes. ${ }^{7}$ This putsenormous economic strain on healthcare expenditure in developingcountries such as South Africa, depriving the state of financial resources that could be better used to address other pressing issues such as HIV AIDS and tuberculosis treatment.However, there is great potential for the use of Information and Communication Technology (ICT) as one of the supporting systems within healthcare.As such, ICT could play an important role in addressing the pressing challenges facing healthcare systems in developing countries, including the issue of medication nonadherence. The recognition of ICT in the health sector is not an end unto itself but a means to an end - and that end is better quality, safer, more value-driven and accessible healthcare to all people. ${ }^{8}$

\section{OBJECTIVES}

The objectives of this paper are to investigate medication adherence among elderly people in retirement villages in South Africa who use a medication regimen of two to four doses per day and, based on the findings, propose a mobile phone adherence monitoring framework (MPAMF) to improve their medication adherence.

The content of this paper is presented as follows: literature, methods, results and discussion, proposed mobile phone adherence monitoring framework (MPAMF)and, finally, the conclusion.

\section{RELATED WORK}

Within the United States and around the world, there is compelling evidence that many patients do not take their medicines as prescribed, resulting into significant consequences. ${ }^{9}$ Medication noncompliance can result in unnecessary disease progression, complications, lower quality of life, and even mortality. ${ }^{10}$ Furthermore medication non-adherence in the elderly results in higher rates of hospital admissions. Other factors associated with a higher risk of hospitalisation in the elderly due to noncompliance includepoor recall of medication regimens, seeing numerous physicians,using numerous medications, and holding the opinion that medications are expensive. ${ }^{11}$

As medical science has made possible new therapies and medicines to effectively treat more chronic or fatal diseases, medication schedules and conflicts between medicines have become more complicated and difficult for general patients to grasp. This problem is even worse for elderly patients who are forgetful or have dementia. ${ }^{10}$

There are numerous potential applications for ICT in a medication adherence intervention. One such application is the use of electronic pharmacy data, kept in hospital servers whichmay allow instances of non-adherence to be identified and may also facilitate delivery of such data to prescribers and pharmacists. ${ }^{12}$ In addition, electronic systems may be able to provide inexpensive remindersabout refills to both patients and providers. Other interactive electronic systems(in other words, ICT applications)could be used to educate patients about appropriate medication use and could also accommodate more sophisticated monitoring, interaction, and communication between the patient and the caregiver. ${ }^{13}$ Although rigorous evaluation of the effectiveness of ICT adherenceinterventions is essential, little systematicresearch into medication adherencethrough the use of ICT (i.e. mobile phone as a tool)in retirement homes has been done.

Mobile health (mHealth) is the use of mobile technology (such as mobile phones)as a delivery tool within thehealthcare sector. MHealth is unique in that it uses a ubiquitous technology (mobile phones)to serve its target populations. It reaches across geographic boundaries and services, is inexpensive and is easy to use andcan be delivered directly to people. Thus it affords a model of care that is both an easily disseminatedand cost effective. mHealth interventions have the capacity to interact with each individual patient, with much greater frequency, and at a convenient time. The use of mHealth has the potential to deliver health behaviour interventions tailored to a person's baseline characteristics such as disease and demographic profile, as well as to take account of frequently changing behaviours and environmental contexts. ${ }^{14}$

In the context of mHealth usage, the individual's perceived self-efficacy is paramount. Perceived self-efficacy is defined as people's beliefs about their capabilities to produce designated levels of performance that exercise influence over events that affect their lives. ${ }^{15}$ In relation to elderly people who are on medication, we interpret perceived self-efficacy as a person's belief that he or she is in control over his or her disease and how well he 
or she manages the disease. The parameters that affect the sense of self-efficacy for patients with chronic conditions include both external and internal attributes. ${ }^{15}$ External attributes include the patient's social network, the health services that are available, as well as financial and IT resources. Such external factors can enhance or impede the effectiveness of a self-help tool. Similarly, internal factors, such as the patient's health status, motivation, level of disease knowledge, and potential functional limitations, also affect his or her sense of self-efficacy and, ultimately, the potential of self-help tools.

\section{METHODS}

In order to achieve the objectives of this paper, the researchercarried out the study in the Gauteng Province of South Africa.Gauteng isSouth Africa's most densely populated province and many retirement homes are situated there. Six retirementhomes were purposefully selected based on their geographical locations spanning the entire province. The retirement homes chosen were all privately owned.The participants for the study were drawn from the entire population of residents in six different retirement homes, each of them over the age of 65 and taking two or more medications three times per day. In describing "population" Polit and Beck indicate that it is the aggregate of cases having a common and designated criterion that is accessible as subjects for a study. ${ }^{16} \mathrm{~A}$ purposive sampling technique was used in selecting the participants. Two elderly people were selected from each of these retirement homes. The participants were selected based on their age (above 65 years) which was relevant to the study. A total of twelve elderly people volunteered to participate in the study. Data was collected using semi-structured open ended interviews. The interviewees were asked to respond to the following questions in their own words:

1. How many different types of medicine do you take per day and how many times per day must you take a dose or doses?

2. What sickness is each medication supposed to treat?

3. How often do you forget to take your medicine per week?

4. Are you reminded by anyone to take your medication when you forget to take it?

5. Do you own a mobile phone?

6. Will you abide by a reminder (e.g.short message or voice prompt) from your mobile phone to take your medication?
The interviews lasted for one-and-a-half hours with each interviewee and were audio-recorded and transcribed by the researcher. Integrity of data entry from the study was checked by another,independent, researcher. Transcripts were codedusing Wolcott's method of case study analysis techniques. ${ }^{17}$ After the initial coding, an independent researcher and the main researcher met to check the consistency of their respective interpretation of the transcripts and the codes. The researcher then coded the final transcripts, identified the main themes, and traced possible relationships. Some broad categories of themes were identified by searching for patterns in the participants' responses. The different broad categories that were noted are discussed below.

\section{RESULTS AND DISCUSSION}

The categories identified were,medication regimensand its treatment effect, external and internal causes of medication non- adherence and medication reminder systems and tools. Each of these categories is discussed below.

\section{Medication regimens and its treatmenteffect}

This section summarises the responses to interview questions 1 and 2 obtained from respondents.

The interviewees indicated that they took an average of four to five pills three times per day. This indicates that, on average, about twelve pillswerebeing taken per person per day. The interviewees further elaborated that their medication was intended to help in the treatment of chronic diseases such as renal disease, coronary artery disease, diabetes mellitus types 1 and 2, hypertension, rheumatoid arthritis and many others. One respondent said:

"Medicationthese daysistoo expensive and taking one pill a day could be good.This will limit me to only two pills per day which I will not even forget to take".

From the responses of the elderly people it may be deduced that adherence to a medication schedule possess a challenge to them but this can be improved by altering the components of the treatment regimen. In such a case, an approach designed to reduce the number of daily administrations of medication by way of using fewer pills designed to be taken once a day, can reduce the complexity of the total regimen. This is supported by Figgewho states that non-compliance of medication adherence in elderly people occurs as a result of their poor recall of the prescribed medication regimen. ${ }^{11}$ This is further echoed by many researchers who statethat the compliance rate can be increased by merely altering the treatment regimen of the patient. 
Arsand and Demiris, however, indicate that it is not only the medicationregimen that affects adherence, but alsothe perceived level of self-efficacy among elderly people. ${ }^{14}$ Perceived self-efficacy is defined as people's beliefs about their capabilities to produce designated levels of performance that exercise influence over events that affect their lives. Related to the elderly people, who are on multiple medications, Arsand and Demiris 2008 interpret perceived self-efficacy as a person's belief in being in control over their disease and how well he manages the disease. The parameters that affect the sense of self-efficacy for patients with chronic conditions include internal attributes such as the patient's health status, motivation level, and level of disease knowledge. ${ }^{14} \mathrm{It}$ is therefore evident that, medication adherence among elderly people is determined not only by the number of pills taken per day, but is also driven by the perceived self-efficacy of the individual patient.

\section{External and internal causes of medication non- adherence}

This section reproduces the responses to questions 3 and 4 , as stated by the interviewees.

Whenthe interviewees were asked to explain how often they forgot to take their medication per week, they indicated that they forgot to take their medicine twice per week on average. They cited the following as the causes of their forgetting to take their medication: forgetfulness due to old age, poor insight into the diseases which were forcing them to take medicine, unknown health status, negative attitude towards medicine, and lack of constant interaction with physicians. Two of the respondents (R1... and $\mathrm{R} 2 \ldots)$ stated

R1: "I forget to take my medicine. I am old and no-one reminds me to take them."

R2: "It makes me dizzy and sometimes I do not take it, especially if I want to go for a walk."

Some of the respondents denied that they were illand therefore saw their medication regimens as an unwelcome idea and something that they did not want to adhere to.

From the responses it was clear that the respondentswould adhere to their medication regimens if they were convinced that the medication they were taking was linked to their future health and wellness, and if they were made an active participant in the decisionmaking process regarding their medication. This means that affected elderly people must be educated about their health, medication and the consequences of nonadherence. It is statedthat interactive electronic systems may be used to educate patients about appropriate medication use, and widespread online connectivity can accommodate more sophisticated monitoring, interaction, and communication. ${ }^{13}$ The family unit and other caregivers can be linked to such an interactive electronic system so as to ensure medication compliance among elderly people. Under such a system, routine automated phone calls and short messages could serve to periodically remind the elderly of their proper medication regimens and dosing times.

It is however, stated that these external and internal causes of non-adherence- which include the patient's social network, the health services that are available, IT resources, the patient's health status and levels of motivation and disease knowledge -are allinfluenced by the individual patient's sense of self-efficacy. ${ }^{14}$

\section{Medication reminder systems and tools}

The respondents were asked if they owned mobile phones and if they would abide by SMS reminders to take their medication. All the interviewees indicated that they owned one or two mobile phones which they used to contact their families when they needed help in obtaining food, money or other items. They further indicated that neither electronic reminders nor SMS were being sent to them to take their medication when they forgot to do so.They reported that only family members would occasionally phone to find out if they were well and if they needed money. One elderly person stated that:

"My cellphone is with me 24 hours. This helps me to communicate with my family and should something happen to me, I will immediately phone them."

It was observed that, despite the high percentage (98\%) of mobile phone ownership among the interviewees, mobile phones were notused to remind the elderly people in the study to abide by their medication regimens.

Based on these findings, the researcher proposes an mHealth application framework which could leverage the existing technologies of mobile phones to remind elderly people to take their medication as prescribed (or remind their caregivers to administer such medication). Such a system would integrate seamlessly into daily routines and would provide alerts when medications should be taken. The mHealth framework proposed in this study would be underpinned bythe perceived self-efficacy of the people using it (which Arsand and Demiris ${ }^{14}$ interpret as a person's belief in being in control over his or her disease and how well he or she manages that disease).

\section{The need for a mobile phone adherence monitoring framework (MPAMF)}

Based on the research findings as presented above, this section proposes a mobile heath framework based on 
self-efficacy for elderly people who live in retirement villages. The proposed mHealth framework would be underpinned by its individual users' sense of self-efficacy and would incorporate:

- reminders to the patient when a medication event is missed

- an escalated reminder service available to approved key caregivers

- warnings when patients take their medication at incorrect times

\section{The diagram below in figure 1 illustrates the MPAMF}

Using a proprietary web-based program, the mobile phone adherence monitoring system would detect noncompliance in real time. Its unique feature set would be able toescalate an appropriate response befitting of the needs of an elderly user and his or her caregivers at a retirement home.

Where such a system is implemented, if the elderly person takeshis medication as prescribed, he logs the event on his mobile phone. This is transmitted to a remote server and, no communication is made to him or her and the "medication event" is stored in the database. On the other hand, if the elderly person does not take his or her medication as prescribed and forgets to log the event on his mobile phone, then he or she is sent a reminder to take the medication.

Moreover, if the elderly person still does not take his medication, then he or she can be reminded again or, if required, a reminder message can be sent to a family member or caregiver advising that the medication has not been taken.

If the person takes the medication outside of the prescribed times, then a message can be sent advising that the medication should not be taken at that time along with a reminder of the when the next prescribed medication event should take place.

The MPAMF can also monitor medication refills and an e-mail message can be sent to the person ora caregiver regarding refill status.

The effective application of the MPAMF is depends on the self-efficacy of the elderly personusing it in each instance.

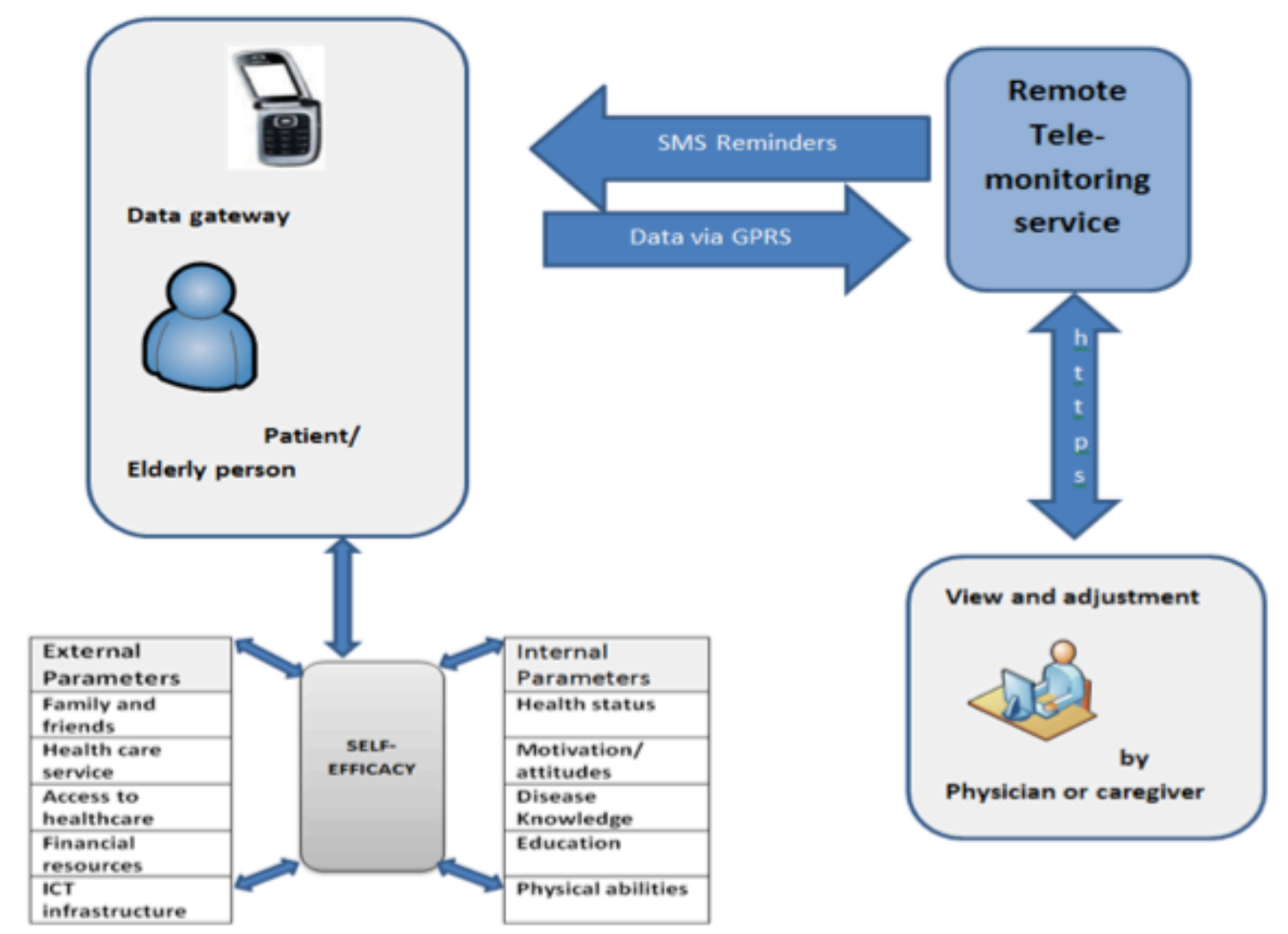

Figure 1: Mobile Phone Adherence Monitoring Framework (MPAMF) 


\section{CONCLUSION}

This paper has reviewed the problems associated with medication adherence among elderly people in retirement homes, unpacked the concept of medication adherence, investigated the causes of non-adherence by elderly people, and has looked into the mHealth tools currently available to elderly people. The research has revealed that elderly peopletake an average of four to five pills, three times per day and that many $(70 \%)$ do not adhere to their medication regimens as prescribed. The research has further revealed that elderly people in the target population own, on average, one or two mobile phones and that these are used to contact family members, but not to receive SMS reminders to take their medication.

Based on the research findings, amobile phone adherence monitoring framework (MPAMF) rootedin the self-efficacy of elderly people living in retirement homes was proposed. The MPAMF is intended to remind elderly people when a medication event is missed, to escalate reminders to approve key caregivers and to send warnings when patients take their medication at incorrect times.

\section{RECOMMENDATION}

It is recommended that:

- elderly people be given initial education regarding the importance of adhering to medication schedules

- basic training be given to the elderly on how messages can be transmitted from and received by mobile phones

- elderly people should be supplied with additional cell phone batteries and chargers

\section{ACKNOWLEDGMENTS}

Mary F Coleman is gratefully acknowledged for her contributionas a lecturer from Medical University of South Africa (Limpopo University) for towards this article.

\section{CONFLICTS OF INTEREST}

Source of Support: Mary F Coleman is gratefully acknowledged for her contribution as a lecturer from Medical University of South Africa (Limpopo University) for towards this article.

\section{ABBREVIATION}

AIDS: Acquired Immune Deficiency Syndrome

HIV: Human Immunodeficiency Virus

ICT: Information and Communication Technology

MHealth: Mobile health

MPAMF: Mobile phone adherence monitoring framework

SMS: Short Message Service

WHO: World Health Organization

\section{REFERENCES}

1. World Health Organization. Adherence to Long-Term Therapies: Evidence for Action. Geneva, Switzerland: World Health Organization. c2003. [Cited 2013 November 11]. Available from: http://whqlibdoc.who.int/ 1984;10: 20-3. publications/2003/9241545992.pdf

2. Osterberg L, Blaschke T. Adherence to medication. N Engl J Med. 2005; 353(5): 487-97.

3. National Community Pharmacists Association (NCPA). Take as Directed: A Prescription Not Followed. Research Conducted by The Polling Company. 2006; December 15

4. Kinsella K, Phillips D.R. Global aging: the challenge of success. Population bulletin. 2005; 60(1): 42.

5. Gray SL, Mahoney JE, Blough DK. Medication adherence in elderly patients receiving home health services following hospital discharge. Ann Pharmacother. 2001; 35: 539-45.

6. Salzman C. Medication compliance in the elderly. J Clin Psychiatry.1995; 56: $18-22$.

7. Einarson TR. Drug-related hospital admissions. Annals of Pharmacotherapy. 1993; 27: 832-40.

8. E-health Initiative and Center for Improving Medication Management. Electronic Prescribing: Becoming Mainstream Practice. A Collaborative Report. From The e-Health Initiative and The Centre for Improving Medication Management. c2008 [Cited 2012 May]. Available from: <www. ehealthinitiative.org.>

9. National Council Report on Patient Information and Education, Enhancing prescription medicine adherence: A national action plan. c2007. [Cited2014, Feb,10]. Available from:http://www.talkaboutrx.org/documents/enhancing prescription_medicine_adherence.pdf.

10. Corey M, Branden M, Zou CC, Zhang NJ. RMAIS: RFID-based Medication Adherence Intelligence System. 32nd Annual International Conference of the IEEE, EMBS Buenos Aires, Argentina; 2010. August 31-September 4.

11. Figge HL. Compliance \& Adherence supplement: US Pharm. 2010; 36: 6-10.

12. Cutrona SL, Choudhry NK, Stedman M. Physician effectiveness in interventions to improve cardiovascular medication adherence: A systematic review. Gen Intern Med. 2010; 25: 1090-6.

13. Kripalani S, Yao X, Haynes R. Interventions to enhance medication adherence in chronic medical conditions: a systematic review. Arch Intern Med. 2007;167: 540-50.

14. Patrick K, Griswold WG, Raab F, Intille SS. Health and the mobile phone. Am. J. Prev. Med. 2008; 35: 177-81.

15. Arsand E, Demiris G. User-centered methods for designing patient-centric self-help tools. Informatics for Health \& Social Care. 2008; 33: 158-9.

16. Polit DF, Beck CT. Nursing research: Generating and assessing evidence for nursing practice. Philadelphia: Lippincott Williams and Wilkins; 2008.

17. Wolcott HF. Transforming Qualitative Data: Description, Analysis, and Interpretation. Thousand Oaks, CA: Sage; 1994.

\section{CONFLICT OF INTEREST}

None Declared 